\title{
No Redundant Error-Correcting Scheme Using Chaotic Dynamics for Noncoherent Chaos Communications
}

\author{
Shintaro $\mathrm{ARAI}^{\dagger}$, Yoshifumi NISHIO ${ }^{\dagger}$ and Takaya YAMAZATO $^{\ddagger}$ \\ ${ }^{\dagger}$ Department of Electrical and Electronic Engineering, Tokushima University \\ 2-1 Minami-Josanjima, Tokushima, 770-8506 JAPAN \\ \{arai, nishio\}@ee.tokushima-u.ac.jp

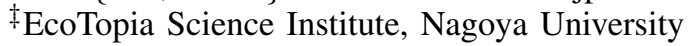 \\ Furo-cho, Chikusa-ku, Nagoya, 464-8603, JAPAN, \\ yamazato@nuee.nagoya-u.ac.jp
}

\begin{abstract}
This paper proposes the error-correcting scheme without redundancy sequences based on the chaotic dynamics for noncoherent chaos communications. We generate successive chaotic sequences from the identical chaotic map. And for the next sequence we set the initial value to the end value of the former sequence. By such way we can create the successive chaotic sequences having the same chaotic dynamics. This feature gives the receiver additional information to correctly recover the received noisy signal. Therefore, by analyzing the chaotic dynamics at the receiver, it is possible to improve the error performance without redundancy signal. As results of computer simulations, we confirm about $3 \mathrm{~dB}$ gain in BER performance as compared with the conventional suboptimal receiver when using the short chaotic sequence length per 1 bit.
\end{abstract}

\section{INTRODUCTION}

Research on digital communications systems using chaos becomes a hot topic [1]- [7]. Especially, it is attracted to develop noncoherent detection systems which do not need to recover basis signals (unmodulated carries) at the receiver. The differential chaos shift keying (DCSK) [1] and the optimal receiver [2] are well known as a typical noncoherent system. In this study, we focus on the optimal receiver.

The optimal receiver calculates the probability density function (PDF) between the received signal and the chaotic maps used on the transmitting side and detects the information symbol by choosing the larger probability. However, the optimal receiver suffers from high computational complexity. Also, using long chaotic sequence decreases the performance of the optimal receiver. Thus, it is important to develop a receiver with performance equivalent to or similar to the optimal receiver using more efficient algorithms, i.e., a suboptimal receiver.

In our previous research, we proposed the suboptimal receiver using the shortest distance approximation [8]. Instead of calculating the PDF, the proposed suboptimal receiver approximates the PDF by calculating shortest distances between the received signal and the chaotic maps and performs detection of the transmitted symbol. As results of the computer simulations, we confirmed the validity of the proposed suboptimal receiver as an approximation method of the optimal receiver.

To improve the performance of the optimal and suboptimal receiver, we are concerned with an error-correcting scheme for noncoherent chaos communications. Especially, we focus on characteristics of chaos to design an error-correcting method. Chaotic sequences obtained from a certain class of difference equations are non-periodic, sensitive to initial conditions and it is difficult to predict their future behavior using past observations. In other words, chaotic sequences are generated according to specific rules, i.e., the chaotic dynamics.

In this study, we focus on the chaotic dynamics and propose a no redundant error-correcting scheme. Let us consider two successive chaotic sequences that are generated from the identical chaotic map. We assume that the second sequence is generated from the initial value which is the end value of the former sequence. In this case, the chaotic dynamics of these two successive chaotic sequences are the same. This feature gives the receiver additional information to correctly recover the received noisy signal. Namely, the error performance of the receiver can be improved by analyzing the chaotic dynamics of the successive received sequences. In addition, as compared with general error correcting methods, our proposed scheme is possible to operate the error-correcting without the physical redundant signal.

We carry out computer simulations and evaluate the performance of the proposed method.

\section{SYSTEM OVERVIEW}

We consider the discrete-time binary CSK communication system with the error-correcting, as shown in Fig. 1.Detail of each block is described below.

\section{A. Transmitter}

In the transmitter, a chaotic sequence is generated by a chaotic map. In this study, we use a skew tent map which is one of simple chaotic maps, as shown in Fig. 2(a), and it is described by Eq. (1)

$$
x_{i+1}= \begin{cases}\frac{2 x_{i}+1-a}{1+a} & \left(-1 \leq x_{i} \leq a\right) \\ \frac{-2 x_{i}+1+a}{1-a} & \left(a<x_{i} \leq 1\right)\end{cases}
$$

where $a$ denotes a position of the top of the skew tent map. The information symbol is modulated by Chaos Shift Keying (CSK) which is a digital modulation system using chaos. When the transmitter generates the signals, we use chaotic sequences generated by different chaotic maps depending on the value of an information symbol. If the information symbol " 1 " is sent, Eq. (1) is used (Fig. 2(a)), and if " 0 " is sent, the 


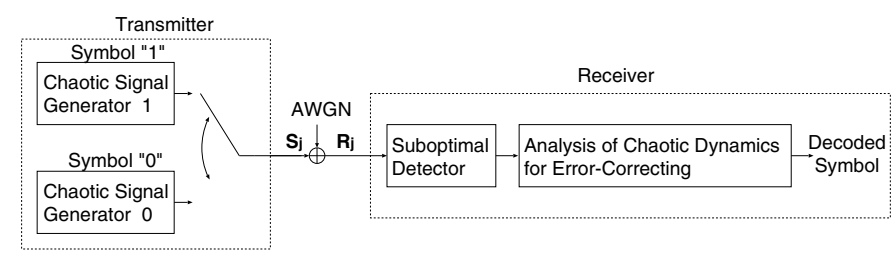

Fig. 1. Block Diagram of Discrete-Time Binary CSK Communication System.

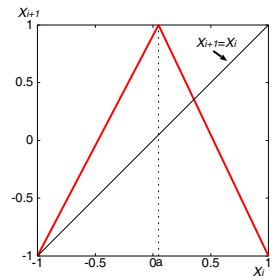

(a) Symbol " 1 "

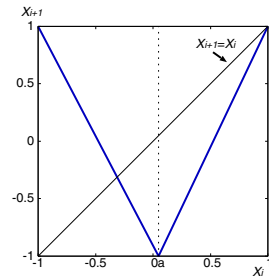

(b) Symbol "0"
Fig. 2. Chaos Shift Keying.

reversed function of Eq. (1) is used (Fig. 2(b)). To transmit a 1-bit information, $N$ chaotic signals are generated, where $N$ is the chaotic sequence length.

In this study, to perform the error-correcting at the receiver, $K$ information bits are transmitted as $K$ signal blocks, as shown in Fig. 3, where $\mathbf{S}_{\mathbf{j}}$ is the transmitted signal vector, $j=(0,1, \cdots, K-1)$, Note that the transmitted signal vector $\mathbf{S}_{\mathbf{j}}$ is different for each symbol.

\section{[When the symbol " 1 " is sent]}

$$
\mathbf{S}_{\mathbf{j}}=\left(x_{\alpha}, f^{(1)}\left(x_{\alpha}\right), \cdots, f^{(i)}\left(x_{\alpha}\right), \cdots, f^{(N-1)}\left(x_{\alpha}\right)\right)
$$

[When the symbol " 0 " is sent]

$$
\mathbf{S}_{\mathbf{j}}=\left(y_{\alpha}, g^{(1)}\left(y_{\alpha}\right), \cdots, g^{(i)}\left(y_{\alpha}\right), \cdots, g^{(N-1)}\left(y_{\alpha}\right)\right)
$$

where $f^{(i)}$ and $g^{(i)}$ are the function of the skew tent map for symbol " 1 " and " 0 " (Figs. 2(a) and (b)) respectively, $i$ is the iteration of $f$ or $g, \alpha=N \times j, x_{j}$ or $y_{j}$ denotes the initial value of the $j$ th symbol $=$ " 1 " or " 0 " respectively. When $K$ bits data is transmitted, the length of the data becomes $K \times N$. An initial value is chosen at random when beginning to make signal blocks and is different in each chaotic signal generator. In addition, the $j$-th sequence is generated from the initial value which is the end value of the former sequence with same symbol of $j$-th bit. As an example, let us assume the $j$ th and $(j+3)$ th symbols are "1" (i.e. the $(j+1)$ th and $(j+2)$ th symbols are " 0 "). In this case, $x_{j+3}=f^{(N)}\left(x_{j}\right)$, and $y_{j+2}=g^{(N)}\left(y_{j+1}\right)$.

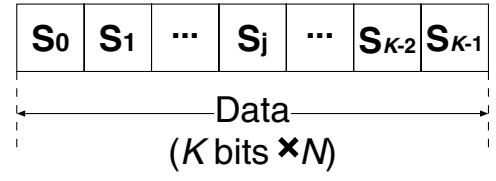

Fig. 3. Signal Blocks.

\section{B. Channel and Noise}

The channel distorts the signal and corrupts it by noise. In this study, noise of the channel is assumed to be the additive white Gaussian noise (AWGN). Thus, the received signals block is given by $\mathbf{R}_{\mathbf{j}}=\left(R_{\alpha}, R_{\alpha+1}, \cdots R_{\alpha+i} \cdots, R_{\alpha+N-1}\right)=\mathbf{S}_{\mathbf{j}}+A W G N$.

\section{Receiver (Suboptimal Detection)}

The receiver recovers the transmitted signals from the received signals and demodulates the information symbol. Also, the receiver performs the error-correcting in this study. Since we consider a noncoherent receiver, the receiver memorizes the chaotic map used for the modulation at the transmitter. However, the receiver never knows the initial value of chaos and the information symbol in the transmitter. Before explaining the error-correcting, we introduce the operation of our suboptimal receiver to be the basis for the proposed error-correcting.

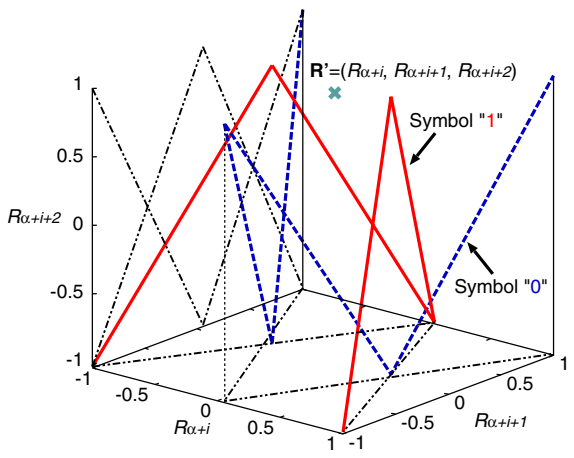

Fig. 4. Operation of Our Suboptimal Receiver $\left(N_{d}=3\right)$.

Our suboptimal receiver calculates the shortest distance between the received signals and the chaotic map in the $N_{d}$-dimensional space using $N_{d}$ successive received signals $\left(N_{d}: 2,3, \cdots\right)$. As an example, we explain the case of $N_{d}=3$. Figure 4 shows the 3 -dimensional space of the skew tent map whose coordinates correspond to the three successive received signals $\mathbf{R}^{\prime}=R_{\alpha+i}, R_{\alpha+i+1}, R_{\alpha+i+2}$. We can calculate the shortest distance between $\mathbf{R}^{\prime}$ and the chaotic map using the scalar product of the vector.

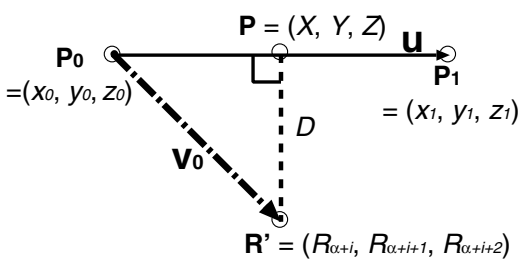

Fig. 5. Calculation of Shortest Distance.

Any two points of $\mathbf{P}_{\mathbf{0}}=\left(x_{0}, y_{0}, z_{0}\right)$ and $\mathbf{P}_{\mathbf{1}}=\left(x_{1}, y_{1}, z_{1}\right)$ are chosen from each straight line in the space of Fig. 4, as shown in Fig. 5. Using Fig. 5, we can calculate $\mathbf{P}=(X, Y, Z)$ and the shortest distance $D$ by the following equations.

$$
\begin{gathered}
\mathbf{P}=(X, Y, Z)=\left(\mathbf{u} \cdot \mathbf{v}_{\mathbf{0}}\right) \mathbf{u}+\mathbf{P}_{\mathbf{0}} \\
D=\left\|\mathbf{P}-\mathbf{R}^{\prime}\right\|
\end{gathered}
$$

where

$$
\begin{aligned}
\text { Unit vector } \mathbf{u} & =\frac{\mathbf{P}_{1}-\mathbf{P}_{\mathbf{0}}}{\left\|\mathbf{P}_{\mathbf{1}}-\mathbf{P}_{\mathbf{0}}\right\|} \\
\mathbf{v}_{\mathbf{0}} & =\mathbf{R}^{\prime}-\mathbf{P}_{\mathbf{0}} .
\end{aligned}
$$

Note that if the point is outside the cube, we calculate the distance between the point and the nearest edges of the maps.

For the 3-dimensional case, there are four straight lines in the space. Therefore, the minimum value in four distances 
is chosen as the shortest distance $D_{1}$ for symbol " 1 ". In the same way, $D$ of symbol " 0 " is chosen as $D_{0}$. We perform these operations until the last sample (i.e., $R_{\alpha+N-1}$ ) is included, and find their summations $\sum D_{1}$ and $\sum D_{0}$. Finally, we decide the decoded symbol as 1 (or 0 ) for $\sum D_{1}<\sum D_{0}$ (or $\left.\sum D_{1}>\sum D_{0}\right)$. The calculation of the shortest distance can be extended to $N_{d}$-dimensional space for $N_{d} \geq 4$.

In our previous research, since we observed that the performance of our method corresponds that of the optimal receiver when $N=N_{d}$-dimension [8], it can be said that the validity of the proposed suboptimal receiver as the approximation method of the optimal receiver.

\section{Proposed ERror-CORRECTING SCHEME WITHOUT REDUNDANCY SEQUENCES}

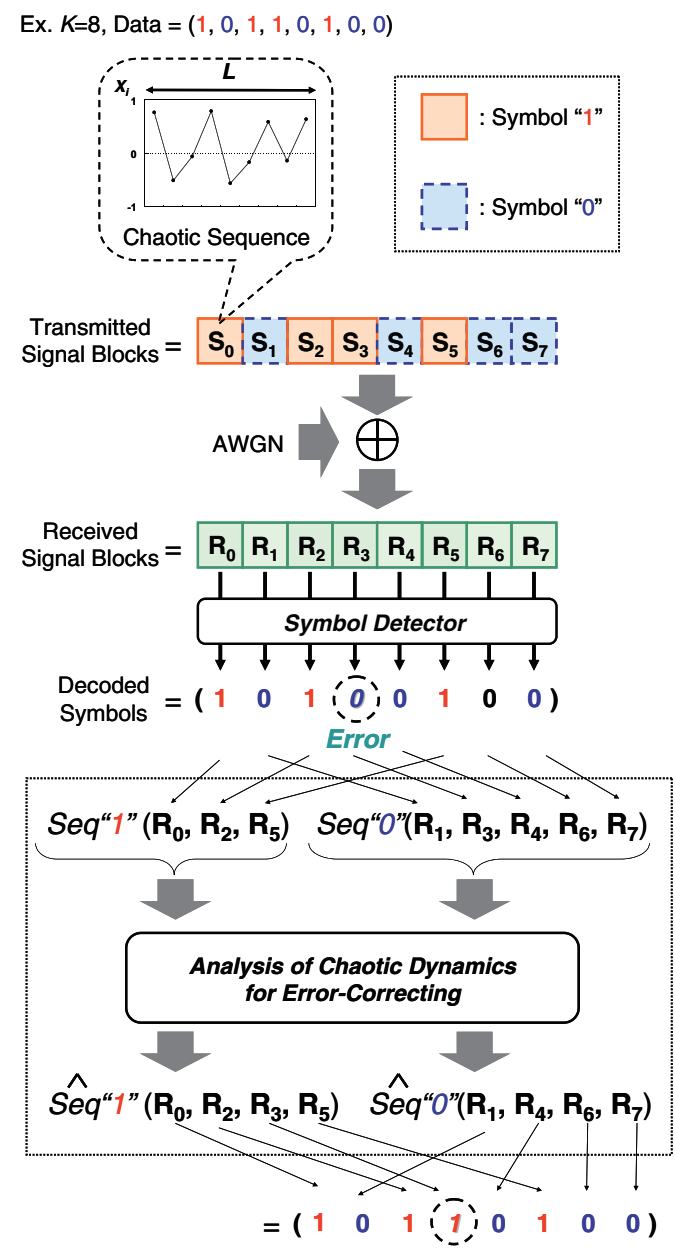

Fig. 6. Operation of Proposed Error-Correcting Scheme).

In this section, we concentrate the proposed error-correcting scheme without redundancy sequences. For ease of explanation, we use Fig. 6 and explain an operation of the proposed error-correcting scheme. Here, we assume $K=8$ and the transmitted symbols $=(1,0,1,1,0,1,0,0)$.

First of all, the receiver performs the noncoherent detection for each received block and demodulates each symbol. In this study, we apply the our suboptimal detection algorithm as the noncoherent detection. Since the transmitted signal blocks arrive at the receiver through AWGN channel. the noise is
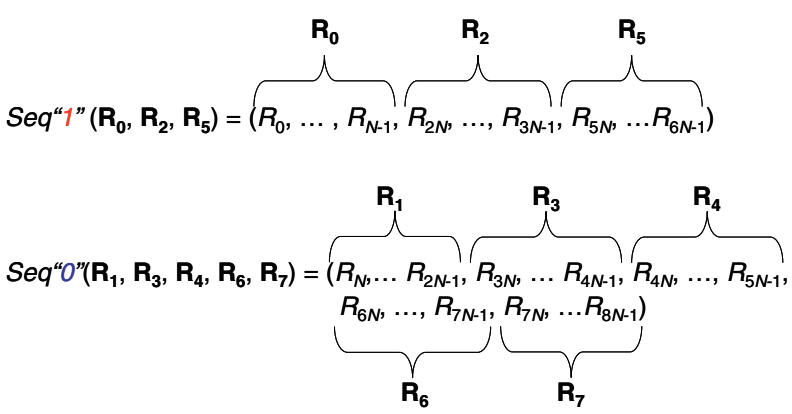

Fig. 7. Sort of Received Signal Blocks according to Decoded Symbols.

$$
D_{\text {Base }}=D_{1}\left(\text { Seq“1" }\left(\mathbf{R}_{0}, \mathbf{R}_{\mathbf{2}}, \mathbf{R}_{\mathbf{5}}\right)\right)+D_{0}\left(\text { Seq“O" }\left(\mathbf{R}_{\mathbf{1}}, \mathbf{R}_{\mathbf{3}}, \mathbf{R}_{\mathbf{4}}, \mathbf{R}_{\mathbf{6}}, \mathbf{R}_{\mathbf{7}}\right)\right)
$$

Reverse

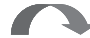

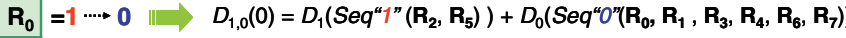

$\mathbf{R}_{\mathbf{1}}=\mathbf{0} \cdots \cdots+1 \| D_{1,0}(1)=D_{1}\left(\operatorname{Seq}^{\prime \prime}{ }^{\prime \prime}\left(\mathbf{R}_{\mathbf{0}}, \mathbf{R}_{\mathbf{1}}, \mathbf{R}_{\mathbf{2}}, \mathbf{R}_{\mathbf{5}}\right)\right)+D_{0}\left(\right.$ Seq $\left.^{\prime \prime} \mathrm{O}^{\prime}\left(\mathbf{R}_{\mathbf{3}}, \mathbf{R}_{\mathbf{4}}, \mathbf{R}_{\mathbf{6}}, \mathbf{R}_{\mathbf{7}}\right)\right)$

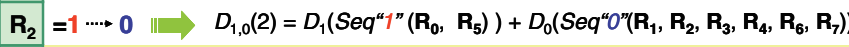

$\mathbf{R}_{\mathbf{3}}=0 \ldots, 1 \| D_{1,0}(3)=D_{1}\left(\right.$ Sequ" $\left."\left(\mathbf{R}_{0}, \mathbf{R}_{\mathbf{2}}, \mathbf{R}_{3}, \mathbf{R}_{\mathbf{5}}\right)\right)+D_{0}\left(\right.$ Sequ" $\left.\left(\mathbf{R}_{1}, \mathbf{R}_{\mathbf{4}}, \mathbf{R}_{6}, \mathbf{R}_{\mathbf{7}}\right)\right)$

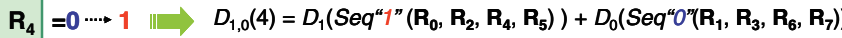

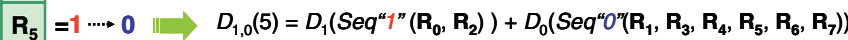

$\mathbf{R}_{6}=0 \cdots \cdots+1 \| D_{1,0}(6)=D_{1}\left(\right.$ Seq $\left.^{\alpha 1}{ }^{\prime \prime}\left(\mathbf{R}_{0}, \mathbf{R}_{\mathbf{2}}, \mathbf{R}_{5}, \mathbf{R}_{6}\right)\right)+D_{0}\left(\right.$ Seq $^{\prime \prime} 0^{\prime \prime}\left(\mathbf{R}_{1}, \mathbf{R}_{3}, \mathbf{R}_{4}, \mathbf{R}_{7}\right)$

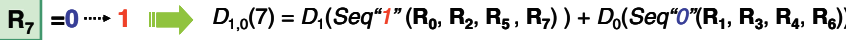

Fig. 8. Analysis of Chaotic Dynamics using Suboptimal Detection.

added to the received signal samples. Thus, the detection error occurs depending on an amplitude of noise. Here, we assume that decoded symbols become $(1,0,1,0,0,1,0,0)$ and the detection error has occurred at $\mathbf{R}_{\mathbf{3}}$.

Next, the receiver sorts the received signal blocks based on decoded symbols and makes two large received sequences; Seq"1"(.) and Seq"0"(.). Figure 7 shows the received sequences which are sorted according to decoded symbols. The reason for sorting blocks is to analyze the chaotic dynamics of the received sequences. In the transmitting side, the chaotic sequences are generated according to transmitted symbols for making the transmitted signal blocks. We have assumed some conditions for an initial value in Seq 2. An initial value is chosen at random when beginning to make signal blocks and is different in each chaotic signal generator. In addition, the $j$ th sequence is generated from the initial value which is the end value of the former sequence with same symbol of $j$-th bit. If the receiver can detects symbols and sorts blocks correctly, we can obtain two large successive chaotic sequences. However, if the detection error is occurred when the receiver detects symbols, the sorted sequence mixes two chaotic sequences which differ in the chaotic dynamics. We focus on these characteristics of chaos for the error-correcting.

For analyzing the chaotic dynamics, the receiver apply our suboptimal detection algorithm, i.e., the calculation of the shortest distance between the chaotic maps and two sorted received sequences. Figure 8 shows conceivable combinations of sorted sequences. Here, $D_{1}($.$) and D_{0}($.$) mean the shortest$ distance between the chaotic map of Symbol "1" and "0", respectively. In addition, we define a reference value for analyzing the chaotic dynamics as $D_{\text {Base }}$. This equation is the sum of the shortest distance between sorted sequences according to symbols which detects as "1" and " 0 " and the 
chaotic map corresponding to their sequences. We also define comparison values to analyze the chaotic dynamics as $D_{1,0}(j)$ $(j=0,1, \cdots, K-1)$. This equation means the shortest distance between sorted sequence when the $j$-th decoded symbol is reversed and the chaotic map corresponding to their sequences. If the receiver can detects symbols and sorts blocks correctly, $D_{1,0}(0) \sim D_{1,0}(K-1)$ become lager values as compared with $D_{\text {Base }}$. On the other hands, if the detection error is occurred, some of $D_{1,0}(0) \sim D_{1,0}(K-1)$ become smaller as compared with $D_{\text {Base }}$. The reason for changing the values is also to change combinations of the chaotic dynamics. Namely, the receiver calculations the shortest distances between some combinations and the chaotic maps and corrects an error. For example, when the detection error has occurred at $\mathbf{R}_{\mathbf{3}}$ as shown in Fig. 6, $D_{1,0}(3)$ becomes the smallest values as compared with $D_{\text {Base }}$ and other other $D_{1,0}(j)$. The receiver can determine that the detection error is occurred at the $3 \mathrm{rd}$ received block.

Therefore, the likelihood of information can improve by analyzing the chaotic dynamics without redundancy sequences, and the receiver can correct an error in high accuracy. Although the error is occurred at one received block only to simplify the explanation, the proposed method is also applicable for more than one error. In this case, after correcting one error, the receiver sorts the received signal blocks according to the decoded symbols again and repeats the same error-correcting operation.

\section{Simulation Results and Discussion}

To evaluate the proposed error-correcting scheme, we carry out computer simulations with following simulation conditions. In the transmitting side, we assume $K=16,32,64$. The parameter of the skew tent map is fixed as $a=0.05$. We use the chaotic sequence length $N=4$ and 8 . In this study, we confirm $N_{d}$-dimensional space to the chaotic sequence length $\left(N_{d}=N\right)$. Based on these conditions, we iterate the simulation 10,000 times and calculate the average of BERs.

Figures 9(a) and (b) show the BERs versus $E_{b} / N_{0}$ for $N=N_{d}=4$ and $N=N_{d}=8$, respectively. To compare the performance of the error-correcting method, Figs. 9(a) and (b) also show the performance of our suboptimal receiver, namely, the performance without the error-correcting. From these results, we observe that the both BERs of the errorcorrecting method show gain over the system without the coding. Significant improvements are observed for $N=N_{d}=$ 4.

As this reason, we consider that it is related to use skew tent map for the chaotic signal generator. Since the skew tent map is the one-dimensional map, a present chaotic signal influences next signal. When a chaotic sequence per 1 bit is short, the influence its initial value on the last value is strong. On the other hand, when a chaotic sequence per 1 bit is large, the influence its initial value on the last value is weak. Therefore, our proposed method with $N=N_{d}=4$ easily analyzes the chaotic dynamics of the sorted sequence as compared with $N=N_{d}=8$. In other words the method using the short chaotic sequence per 1 bit is more suitable for our errorcorrecting than using long sequence. This characteristic is different from the maximum-likelihood decoding. We can say that these results are caused by unique characteristic of chaos.

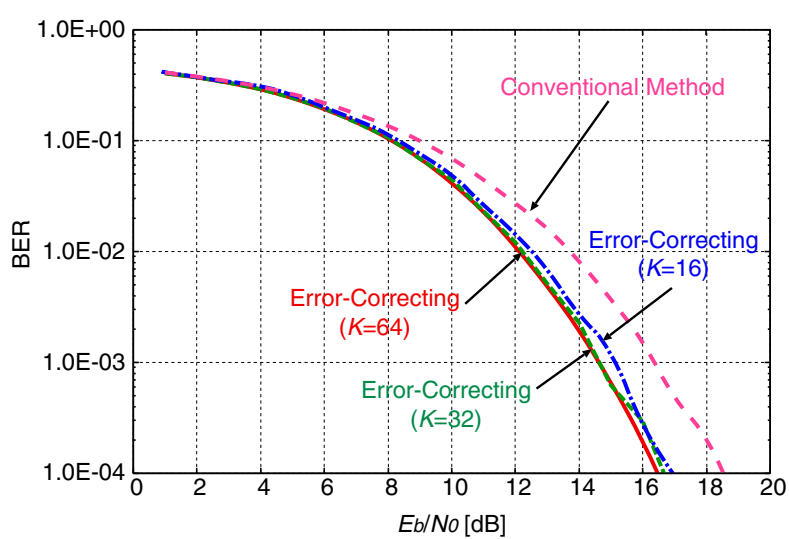

(a) $N_{d}=4$.

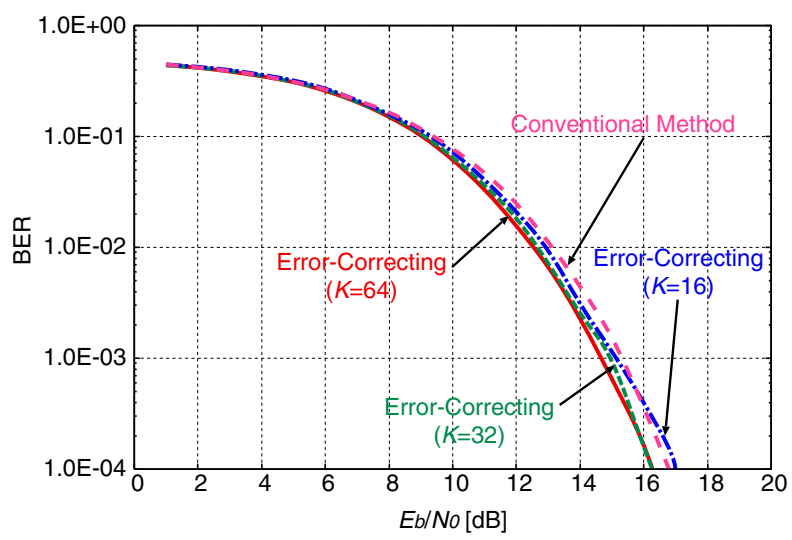

(b) $N_{d}=8$.

Fig. 9. Simulation Results.

\section{Conclusions}

In this study, we have focused on the chaotic dynamics and proposed the error-correcting scheme without redundancy sequences. As results, we have confirmed that the likelihood of the received symbol increases and obtained the $2-3 \mathrm{~dB}$ gain in error performance of the proposed method with $N=N_{d}=4$.

\section{REFERENCES}

[1] G. Kolumbán, B. Vizvári, W. Schwarz, and A. Abel, "Differential chaos shift keying: A robust coding for chaos communication," Proc. NDES'96, pp. 87-92, Jun. 1996.

[2] M. Hasler and T. Schimming, "Chaos communication over noisy channels," Int . Journal of Bifurcation and Chaos, vol. 10, no. 4, pp. 719-736, Apr. 2000.

[3] M. Hasler and T. Schimming, "Optimal and suboptimal chaos receivers," Proc. IEEE, vol. 90, Issue 5, pp. 733-746, May 2002.

[4] F. C. M. Lau and C. K. Tse, Chaos-Based Digital Communication Systems, Springer, 2003.

[5] W. M. Tam, F. C. M. Lau and C. K. Tse, "Generalized correlation-delayShift-Keying Scheme for Noncoherent Chaos-Based Communication Systems,' IEEE Trans. Circuits and Systems Part I, vol. 53, no. 3, pp. 712721, Mar. 2006.

[6] L. E. Larson, J-M. Liu, L. S. Tsimring, Digital Communications Using Chaos and Nonlinear Dynamics, Springer, 2006.

[7] G. Cimatti, R. Rovatti and G. Setti, "Chaos-Based Spreading in DS-UWB Sensor Networks Increases Available Bit Rate," IEEE Trans. Circuits and Systems Part I, vol. 54, no. 6, pp. 1327-1339, Jun. 2007.

[8] S. Arai and Y. Nishio, "Detection of Information Symbols and Sequence Lengths Using Suboptimal Receiver for Chaos Shift Keying," Proc NOLTA'06, pp. 799-802, Sep. 2006. 